\title{
Zwischen Tradition und Moderne: persönliche Erfahrungen und Erkenntnisse nach sieben Jahren in der dänischen Erwachsenenbildung
}

\author{
Von Martin Groh
}

In Vartov, Kopenhagens ehemaligem Hospital und Armenstift aus dem 18. Jahrhundert, befindet sich die Grundtvig-Bibliothek. Sieht man aus einem ihrer Fenster erhält man einen interessanten Anblick. Fast drohend erhebt sich der Turm des Rathauses über die Dächer Vartovs. So nah, als würde er sich jeden Augenblick über den kleinen Nachbarn stürzen wollen, wirkt er in seiner Erhabenheit gleichzeitig sehr fern. Der Architekt Martin Nyrop setzte den mächtigen Rathausbau 1892-1905 als unübersehbares Zeichen für das beginnende Zeitalter der modernen und demokratischen Industriegesellschaft auf Kopenhagens ehemalige Wälle. Die Rathausuhr, deren Glocken jede Viertelstunde die Zeit angeben, fügt diesem optischen Symbol den akustischen Takt hinzu. Ihr Klang ist allen Dänen bekannt und sie bestimmt bis heute unwillkürlich den Rhythmus der Menschen, die in der Nähe des Rathauses arbeiten und leben.

Einen ganz anderen Eindruck dagegen vermittelt Vartov. Das barocke Gebäude umschließt einen großen Hof, dessen Stille jeden völlig überrascht, der die Hektik der umgebenden Straßen verläßt und durch die großen Tore eintritt. Beim Anblick des alten Pflasters, der schlichten, aber sehr wirksamen Architektur, des Glockengestells, der Lindenbäume und Ruhebänke hat man den fast unwirklichen Eindruck einer Reise in die Vergangenheit. Mitten in dieser Idylle steht eine Skulptur, die N. F. S. Grundtvig darstellt. Es ist eine Arbeit des Künstlers Niels Skovgaard, der hier ein Paradox geschaffen hat. Die Bronzeskulptur ist nämlich überlebensgroß und zusätzlich auf einen massiven Sockel gestellt. Dieser Eindruck von Größe und Dominanz wird aber dadurch gemildert, daß Grundtvig in einer knienden Pose, mit leicht gesenktem Kopf und offenen Händen dargestellt ist. Eine Geste, die Demut symbolisiert und so gar nicht zu dem Bild des mächtigen und selbstbewußten Patriarchen Grundtvig paßt, das ansonsten in der Öffentlichkeit verankert ist. Ein Bild, das auch ich in den sieben Jahren meiner Bekanntschaft mit dieser historischen Gestalt und ihrem Nachleben verinnerlicht habe. 
Vartov beherbergt heute neben vielen anderen Institutionen den Hauptsitz von Kirkeligt Samfund und ist ein wichtiger Ort für die Grundtvigsche Bewegung in Dänemark. Dafür sorgt die hier plazierte Grundtvig-Bibliothek, die den größten Teil seiner Werke und der Literatur darüber enthält, und die GrundtvigAkademie, die hier Anfang 1999 etabliert wurde. Aber auch Verbände der Freien Schulen haben in Vartov ihren Sitz, beziehungsweise halten dort ihre Treffen ab. Die Säle und Räume des Hauses bieten den Rahmen für Veranstaltungen von allen gesellschaftlichen Gruppen und Organisationen in Dänemark, die den Gedanken Grundtvigs nahestehen oder sich mit seinem Wirken beschäftigen wollen. Die Atmosphäre Vartovs wirkt noch authentischer dadurch, daß sich in seinen Mauern die Kirche befindet, in der Grundtvig über 30 Jahre seines Lebens gepredigt hat. Das Haus repräsentiert auf diese Weise das Universum, in dem sich meiner Meinung nach der Grundtvigianismus heute bewegt: Kirche und christlicher Glaube, wissenschaftliches Studium der Ideen Grundtvigs und der Versuch, diese Ideen vor allem über die lebenslange Bildung - oder sollte man hier besser den dänischen Begriff der »folkelig livsoplysning « benutzen - den Dänen immer wieder von neuem zu vermitteln.

Vor allem in diesem letzten Bereich, der dänischen Erwachsenenbildung liegen meine persönlichen Erfahrungen mit dem Grundtvigianismus. Von der Kieler Universität kommend, wurde ich 1993 an der dänischen Højskolen Østersøen in Aabenraa als Lehrer für Deutsch und Geschichte angestellt. Die Schule war eine Neugründung und die Aufgabe des neuen Kollegiums bestand zu einem großen Teil darin, neben der Gestaltung des Unterrichts dem Gedanken der Kostschule eine reale Form und einen täglichen Rhythmus zu geben.

Hier ergab sich bereits ein wesentliches Problem, da nämlich die Hälfte der Lehrer Deutsche waren, für die sich eine fast unbekannte Welt auftat. Aber auch das Wissen der dänischen Kollegen um Inhalte und Bedeutung der KostschuleDimension schien auf eher unzusammenhängenden Kenntnissen zu beruhen. Ohne es empirisch belegen zu können, behaupte ich hier eine generelle Tendenz zu erkennen, die mir wiederholte Beobachtungen an anderen folkehøjskoler und der Meinungsaustausch mit dortigen Kollegen bestätigt haben. ${ }^{1}$ Viele højskoleLehrer haben nur oberflächliches Wissen über Grundtvig und die Geschichte ihrer Schulform. Sie zeigen oft aber auch nur geringes Interesse am ideologischen Unterbau ihrer Schultradition und überlassen dieses Feld den Schulleitern. Die Vorsteher erhalten dadurch die priviligierte Rolle der Erbträger des 
Wissens um die wahre Bedeutung der Grundtvigschen Formel von "støv og ånd", der Verbindung von materiellem Dasein und geistigem Wirken.

1994, im 150. Jubiläumsjahr der folkehøjskoler, machte sich diese Tatsache bemerkbar. Die damals begonnene generelle Debatte um eine Neudefmition der folkehøjskoler und der Freien Schulen wurde hauptsächlich von bestimmten Schulleitern und Repräsentanten der fachlichen Organisationen geführt. ${ }^{2} \mathrm{Die}$ Diskussion bewegte sich auf zwei Ebenen, die auf den ersten Blick nichts miteinander zu tun hatten. Zum einen ging es um Inhalte von Lehre und Form und um das Verhältnis der Freien Schulen, und hier besonders der folkehøjskoler, untereinander. Die zweite Diskussionsebene aber beinhaltete die Frage nach dem Status und der Rolle der Freien Schulen in der dänischen Gesellschaft. Diese existentielle Frage wurde durch das neue Gesetz für die Freien Schulen aufgeworfen, das der damalige Unterrichtsminister Ole Vig Jensen auf der Generalversammlung in Ryslinge am 20. August 1993 vorstellte. ${ }^{3}$ In seiner Rede verwies der Minister auf die hohe Zahl von Jugendlichen, die nur schwer in Ausbildungsplätze zu vermitteln sei oder Lehrgänge vorzeitig abbreche. Er kündigte entsprechende Gegenmaßnahmen der Regierung an und die Absicht, die folkehøjskoler als Hilfe bei der Beseitigung dieser Mißstände in die Pflicht zu nehmen.

Ole Vig Jensen nannte in seiner Argumentation zwei zentrale Begriffe: "folkelig livsoplysning «, was man als "Volksbildung zum Leben« übersetzen könnte, und »folkets almindelige anliggender«, auf deutsch »die allgemeinen Anliegen des Volkes«. Unter dem ersten Begriff verstand er die Aufgabe der Freien Schulen, über die gesellschaftlichen Zusammenhänge (»folkelige fællesskaber«) aufzuklären und Möglichkeiten zur Erläuterung des Daseins (॥tolkning af tilværelsen«) zu geben. Dies sollte einerseits durch allgemeinbildenden Unterricht und andererseits durch Gemeinschaft geschehen. Für den Minister war es auch wichtig, die Freien Schulen besser mit ihrem nichtfachlichen Hinterland zu verbinden, das heißt, das Verhältnis der Schulen und ihrer Vorsteher zu den Vorständen und Schulvereinen neu zu formieren.

Der Begriff der »allgemeinen Anliegen des Volkes« ist eine bemerkenswerte Formulierung, wobei ich "Anliegen« als "Interessen" des Volkes verstehe. Ole Vig Jensen zielte hier meiner Meinung nach deutlich auf die direkten Anforderungen der dänischen Gesellschaft an den Allgemeinnutzen vor allem der folkehøjskoler. Die folkehøjskoler seien zwar nicht Teil des formellen Aus- 
bildungssystems und sollten dies auch nicht werden, aber die mangelnden Ausbildungsmöglichkeiten für Jugendliche seien auch ihr Problem. Mit dieser Aussage begab sich der Minister als Vertreter des Staates auf das Terrain des Gegenübers, indem er das ureigenste Prinzip der Freien Schulen berührte, das Prinzip der »Freiheit « in Form, Lehre und Organisation. Dessen war sich Ole Vig Jensen selbstverständlich bewußt. Er beeilte sich daher, zweimal an anderer Stelle seiner Rede die Freiheit der Freien Schulen zu betonen.

Das Prinzip der Freiheit wàr grundlegend für Grundtvigs gesamte Gedankenwelt. In seinem berühmten Programmgedicht $»$ Freiheit für Loke wie für Thor « in »Nordens Mythologi« von 1832 konzipierte er seine Idee von Freiheit als den Ausdruck des freien individuellen Willens, der in einem dauernden "Kampf" der unterschiedlichen Meinungen in Politik, Religion, Bildung und Wissenschaft überhaupt erst zu einer freien Gesellschaft führe. Ein immer wiederkehrendes Bild, zum Beispiel in den »Mands Minde«-Vorträgen, war seine Ansicht von der freien Gesellschaft als ein lebendiger Organismus, der sich durch die Konkurrenz der Individuen untereinander ständig in Entwicklung befinde. Diese Entwicklung konnte aber nur funktionieren, wenn sich die Menschen als »von gleichem Wert « verstanden. Grundtvig erkannte, daß der Staat immer wieder versuchen würde, die freiheitliche Entwicklung zu kontrollieren und notfalls zu begrenzen. Ihm war aber gleichzeitig klar, daß der Staat den Rahmen bildete, der einen friedlichen und geregelten Verlauf des Freiheitsprozesses garantierte. ${ }^{4}$

Der freie Wille des Einzelnen, für das »fælles bedste « - das "gemeinsame Beste« - Verantwortung übernehmen zu wollen, war Grundtvig zufolge der Grundstein für die Bildung einer »folkefællesskab«, einer »Gemeinschaft des Volkes «. ${ }^{5}$ Diese Definition von Volk ließ zunächst viele Möglichkeiten offen. $\mathrm{Da}$ Grundtvig sie aber an das Sprechen einer gemeinsamen Muttersprache knüpfte, wurde sie im Zeichen des beginnenden Nationalitätenkampfes zwischen deutsch und dänisch in den 1840er Jahren schnell zu einer exklusiven, den anderen im Sinne dieses Wortes ausschließenden Bestimmung.

Für viele der ersten, zwischen 1842 und 1850 gegründeten højskoler war die nationalpolitische Zielsetzung zweitrangig. Sie verstanden sich eher als unabhängige, private Bauernschulen, in denen die vom öffentlichen Schulsystem oft benachteiligte Landjugend versäumtes Wissen nachholen konnte. Gleichzeitig sollten die Schüler zu mündigen und »freien« Bürgern erzogen werden. Dem- 
entsprechend gering war der Einfluß der nationalen Gedanken Grundtvigs in den Anfangsjahren der Freien Schulen. ${ }^{6}$ Erst mit der Niederlage gegen Preußen 1864 entwickelten sich diese Schulen zu folkehøjskoler, deren vornehmste Aufgabe nun im Aufbau einer nationalstaatlichen Identität bei der gesamten dänischen Jugend bestand. Die seit den 1870ern praktizierte Neutralität, die Dänemark allmählich in eine immer größer werdende internationale Isolation führte, war in Wirklichkeit die nach außen gerichtete Entsprechung des eng gefaßten dänischen Nationalitätsbegriffes. Die folkehøjskoler spielten hier eine wesentliche Rolle und profitierten, von einigen konjunkturellen Schwankungen abgesehen, von dieser Zielsetzung.

Nach fünf Jahren Besatzung durch die Deutschen war den Dänen 1945 bewußt geworden, daß der Weg der Neutralität fast zum Zusammenbruch ihrer Nation geführt hätte. Der neue Weg bestand in der Aufgabe der Neutralität durch den Beitritt zu UNO und NATO und schließlich die politische und ökonomische Öffnung des Landes zur Europäischen Gemeinschaft seit 1972. Diese Entwicklung stellte die folkehøjskoler allmählich vor ein Dilemma, das sie meines Erachtens bis heute nicht gelöst haben. Das im Gegenteil durch die zunehmende Internationalisierung seit den 1990er Jahren zu einem existentiellen Problem für die dänische Erwachsenenbildung geworden ist. Denn das "gemeinsame Beste« läßt sich schon seit 1945 nicht mehr unbedingt als ein Anspruch interpretieren, der exklusiv den Dänen vorbehalten ist.

150 Jahre lang war das Prinzip der Freiheit im Sinne Grundtvigs die Wurzel für die Existenzberechtigung der folkehøjskoler als gesellschaftliche Institution. Der dänische Staat ließ sie als Alternative zum öffentlichen Schulsystem gewähren, ja unterstützte sie moralisch und finanziell, weil er sie brauchte. Er brauchte sie, um den Gedanken der »folkelighed «, der »Volksnation«, in weiten Teilen der Bevölkerung verankert zu wissen. Bedeutet dies, daß sich die folkehøjskoler seit 1864 von der Politik instrumentalisieren ließen, nur weil sie dafür einen in der ganzen Welt einmaligen Grad an Freiheit erhielten? Keineswegs - diese Aufgabenteilung verhinderte nicht, daß die folkehøjskoler und auch die anderen freien Schulen oft in Opposition zum herrschenden politischen Denken standen. Aber der Konsens, den man mit dem Staat erreicht hatte, wurde akzeptiert. Für das gemeinsame Beste im Sinne der Interessen der Allgemeinheit zu arbeiten, fand als grundlegender Ethos der Freien Schulen über viele Jahrzehnte trotz wechselnder politischer Vorzeichen immer wieder breite Aner- 
kennung - in der Bevölkerung, bei den »Volksaufklärern« selbst und bei den Politikern.

Heute, am Beginn des 21. Jahrhunderts, müssen die Dänen aber erkennen, $\mathrm{da} ß$ sowohl aus dem Inneren ihrer Gesellschaft, wie von außen die Grundlagen der dänischen Nationalstaatlichkeit erheblich in Frage gestellt werden. Die Einwanderung von Ausländern, die wachsende Mobilität der Arbeitsmärkte, die zunehmende Individualisierung und das umfassende Abtreten von nationalen Befugnissen an internationale Organisationen sind nur einige der Faktoren, die eine neue Beurteilung des Wesens der dänischen ॥Volksnation « bedingen. Hier ist also die ethische Grundlage der folkehøjskoler direkt betroffen. Da für die folkehøjskoler mit der Erfüllung dieser Aufgabe aber auch ihre Freiheit, also ihre institutionelle Grundlage verbunden ist, müßten sie und auch die übrigen Freien Schulen reagieren und eine aktive Rolle in der Neudefmition von »folkelighed « übernehmen. Mein Eindruck während der vergangenen sieben Jahre ist, daß sich ein großer Teil der Freien Schulen gegen diese offensichtliche Entwicklung wehrt und ein weiterer Teil sich passiv und abwartend im Nischendasein verhält. Nur ein verhältnismäßig kleiner Teil hat die seit 1996 sinkenden Teilnehmerzahlen wirklich zum Anlaß genommen, einen Denkprozeß in Gang zu setzen und dafür die fachlichen Organisationen der Freien Schulen zu gewinnen.

Wesentlicher Grund für diese unterschiedliche Haltung ist meiner Ansicht nach, daß sich das Prinzip der Freiheit mehr und mehr als Hemmnis für ein gemeinsames Handeln herausstellt. Freiheit wird zu sehr als individuelle Freiheit betrachtet. In Situationen, die für alle schwierig sind, streben die Freien Schulen nicht nach Einigkeit, sondern beharren zu sehr auf ihrem Recht auch voneinander unabhängig zu sein. Um das zu erläutern, komme ich zurück zum Jubiläumsjahr 1994 und der ersten Ebene der generellen Debatte, die damals unter dem Schlagwort $» K e t z e r$ gegen Orthodoxe» geführt wurde. Sie betraf das Verhältnis der folkehøjskoler untereinander und die Frage danach, was wahres højskole-Wirken im Sinne Grundtvigscher Traditionen sei, oder was sich nur unter dem Etikett der Kostschule tarne und in Wirklichkeit reines Ausnutzen staatlicher Zuschüsse bedeute.

Im Kern handelte es sich aber um die Frage nach Ziel und Zweck der pädagogischen Grundlage der Freien Schulen und besonders der folkehøjskoler: der "folkelig livsoplysning«. Ole Vig Jensen hatte, wie bereits erwähnt, schon 1993 
definiert, was er darunter verstand. Seine Formulierungen von der »Aufklärung über die gesellschaftlichen Zusammenhänge« und der »Auslegung des Daseins« waren natürlich bewußt offen und vage formuliert. Morten Kvist, Urheber des "Ketzer-Wortes", konnte hingegen viel konkreter werden. Für ihn bestanden die unverzichtbaren Ecksteine der højskole aus »Examensfreiheit«, »sorgfältiger Sprachpflege«, »historischem Bewußtsein« und »Singen.$^{7}$ Viele weitere Stimmen nannten in den folgenden Jahren viele weitere Definitionsansätze und goldene Regeln für die »richtige« beziehungsweise "falsche» Schule. Keine kam wirklich zu einer endgültigen, allgemein akzeptierten Lösung.

Persönlich war ich damals als neuer, unerfahrener und anfangs recht desorientierter Lehrer sehr daran interessiert, eine solche Navigationstabelle in die Hand zu bekommen. Meine Verunsicherung wurde durch die Tatsache gesteigert, daß die Højskolen Østersøen seinerzeit in das Ketzer-Lager eingeordnet wurde, weil sie mit zwei Tabus brach. Sie suchte engen Kontakt zur Privatwirtschaft und hatte mit dem Schwerpunkt Deutschunterricht einen gewollt starken fachlichen Bezug. Heute bin ich überzeugt, daß es gar keine endgültige Definition für die alltägliche Verwirklichung von »folkelig livsoplysning « gibt. Die Debatte wurde vielmehr benutzt, um in einer Zeit größer werdender Konkurrenz und mit einer zunehmend kritischen Öffentlichkeit einzelnen Positionen frühzeitig eine gute Ausgangslage zu verschaffen. Sie verdeckte dadurch eine Diskussion über den wirklichen Inhalt und den Ursprung dieses Grundtvigschen Begriffes.

Grundtvig hatte 1834 in seiner Schrift »Den danske Stats-Kirke upartisk betragtet ( (»Die dänische Staats-Kirche unparteiisch betrachtet «) bereits vorweggenommen, was er in seiner zentralen pädagogischen Programmaussage »Statsmæssig Oplysning " aus dem selben Jahr als Hauptthemen für eine "staatsmäßige Aufklärung « ansah: Die Rücksicht auf das Wohl des Staates, die Warnung vor einem übertriebenen Individualismus und die Notwendigkeit, eine "Volksakademie« einzurichten. Vom Begriff der staatsmäßigen Aufklärung ging Grundtvig dann im Laufe der nächsten beiden Jahre dazu über, von der »Folkelig Oplysning « oder der »Livs-Oplysning « zu sprechen. ${ }^{8}$ Die drei Elemente »folkelig«, »liv« und »oplysning» beschreiben den Kern der Grundtvigschen Aufklärungsphilosophie. Aufklärung sollte über und für das von Gott gegebene Leben erfolgen. Sie sollte dem Individualismus, wie ihn die Kantsche Vernunftphilosophie predigte, entgegenwirken, indem sie die mitmenschliche 
Gemeinschaft förderte und gleichzeitig dem ganzen Volk offenstand. Die »Lebensaufklärung" sollte dabei nicht wie ihr Gegenpart, die klassische, buchorientierte »Papirs-Oplysning«, Selbstzweck sein, sondern den Schülern durch das »Erleben « der Gemeinschaft Lebensfreude vermitteln. ${ }^{9}$

Dabei lehnte Grundtvig die Fachlichkeit keineswegs ab. Es kam ihm viel mehr darauf an, daß die Schulen sich zur Lebensaufklärung als dem generell höheren Ziel und als der Richtschnur für jedes einzelne Fach bekannten. Der einzelne Lehrer sollte diesem Grundsatz folgen, indem er mit den Schülern in einen Dialog trat. Wichtige Voraussetzung für eine solch »lebendige Wechselwirkung « zwischen Lehrer und Schüler war die Examensfreiheit. ${ }^{10}$ Gerade in der seit 1996 aufgetretenen Krisensituation, die bereits einige folkehøjskoler die Existenz gekostet hat, ist auch immer wieder über die Einführung von Examen in den folkehøjskoler diskutiert worden. Auf diese Weise sollten die folkehøjskoler auf dem Papier beweisen können, daß sich die Qualität ihres Unterrichtes mit dem des öffentlichen Schulsystems sichtbar messen lassen kann.

Heute ist der Alltag vor allem junger Menschen immer mehr von moderner Computertechnologie geprägt. Diese Technologie bedeutet, daß Jugendliche von einem Umfeld umgeben sind, das sich an sichtbarem Funktionieren oder Nicht-Funktionieren orientiert. Eine wachsende Informationsmenge steht ihnen zur Verfügung, ohne daß die Möglichkeiten sich in dieser Vielfalt zu orientieren, in gleichem Maße wachsen. In der Wissensgesellschaft wird dadurch das Angebot an Ausbildung durch die sogenannte »kompetencegivende uddannelse« wichtiger. Hiermit ist die formalisierte Ausbildung gemeint. Sie erteilt Kompetenzen, oder Qualifikationen, nach schematisierten, von vornherein festgelegten und dadurch nachweisbaren Leistungszielen - ganz nach dem Motto »funktioniert oder funktioniert nicht «. Die folkehøjskoler hingegen müssen immer größere Anstrengungen unternehmen, um ihre Klientel vom Nutzen der lebenslangen Bildung als Gegenmodell zur formalisierten Ausbildung des öffentlichen Schulsystems zu überzeugen. Ein Grund ist sicher, daß es in der rationalisierten Gesellschaft schwieriger geworden ist, die sogenannten "weichen« Qualifikationen, Grundtvigs Überzeugungen von Gemeinschaft, Erleben und Lebensfreude zu vermitteln. Aber für sich genommen, genießen diese Werte immer noch eine hohe Akzeptanz.

In den letzten dreißig Jahren haben die folkehøjskoler meiner Meinung nach einen großen Teil ihres Verständnisses für die gesellschaftliche Realität verlo- 
ren, und das obwohl ihre Schülerschaft diese Realität widerspiegelt. Die Debatte über die Bedeutung der »folkelig livsoplysning" wurde nicht geführt, um sich über die Ursprünge der gemeinsamen Traditionen klar zu werden, sondern um zu polarisieren. Die Grundtvigsche Bewegung nimmt zu Recht für sich in Anspruch, von ihrem Beginn an wesentlicher Garant für eine friedliche, demokratische und stabile Entwicklung der dänischen »folkefællesskab « gewesen zu sein. Teile dieser selben Bewegung verschließen sich aber einer gemeinsamen, aktiven Politik für das »fælles bedste« ihrer eigenen Schulform. Trotz erkennbarer, massiver Veränderungen ihres gesamten Umfelds und ihrer Wertegrundlagen ziehen sie sich stattdessen auf ideologisch fundierte Bastionen zurück.

Der Staat hat den folkehøjskoler einen großen Freiraum gegeben, und die Schulen haben ihre Freiheiten immer wieder erfolgreich gegen staatliche Begrenzungsversuche verteidigt. Das Verhältnis funktionierte auch deswegen so reibungslos, weil über eine lange Zeit wichtige Politiker Dänemarks aus der Schülerschaft, aber auch aus den Lehrerkollegien und Leitungen der Freien Schulen stammten. Um es populär zu sagen: »man wußte, wo man einander hatte«! Diese Verbindungslinie ist schon seit den 1980er Jahren langsam dünner geworden. Die Konsequenz ist, daß die Lobbyarbeit gerade in der Krisenzeit schwieriger geworden ist. Ein weiteres Problem sehe ich darin, daß die Lehrer in den Freien Schulen zu wenig über die Inhalte und die Geschichte des Grundtvigianismus wissen. Ich meine damit nicht, daß das Auswendiglernen von Fakten über Grundtvigs Wirken die højskole-Lehrer zu besseren Pädagogen macht. Aber weiter und tiefer verbreitete Kenntnisse könnten die existentielle Debatte über die Zukunft der Freien Schulen einem größeren Kreis von Betroffenen öffnen und vom Geruch der Elitediskussion zwischen Vorstehern, Vorständen und Vertretern der fachlichen Organisationen befreien.

Es ist nicht der richtige Weg, die äußere und die innere Freiheit, die Vielfalt innerhalb der Freien Schulen und die Unabhängigkeit gegenüber den öffentlichen Schulen, aufzugeben. Anpassung an das öffentliche System, zum Beispiel durch Einführung von Examina, wäre ein falscher Schritt. Die folkehøjskoler müssen dafür argumentieren, daß es weiterhin wichtig ist, fachlich qualifizierten Unterricht unter das Motto der "folkelig livsoplysning" zu stellen. Weiche Qualifikationen, soziale Kompetenzen und gemeinschaftliches Erleben kombiniert mit fachlich hohem Niveau entsprechen sowohl Grundtvigscher Tradition, als auch dem Anforderungsprofil moderner Wirtschaftsunternehmen. 
Die Aufgabe der Freien Schulen besteht darin, ihre Stärken besser zu vermarkten. In den letzten Jahren sind in dieser Richtung viele Anstrengungen unternommen worden. Aber verstärkte Lobbyarbeit, verstärktes Marketing und sporadische Versuche der Neudefmition werden im Sand verlaufen, wenn sie nicht von einer weitestgehend einigen Stimme vorgebracht werden.

Frederik Christensen hielt 1995 zum Abschluß einer Konferenz der folkehøjskoler in Sønderborg eine Rede zum Thema »den rigtige højskole«. Dabei verteidigte er die oben angeführte Kombination von Tradition und Moderne. Er berief sich auf die großen Vorbilder Kold, Flor und Schrøder, die diese Verflechtung praktiziert hätten, ohne - und dies war das Entscheidende - die mitmenschliche Gemeinschaft als oberstes Ziel aus den Augen zu lassen. Er sagte aber auch, daß der Streit zwischen den folkehøjskoler um die Richtigkeit dieser Ziele wichtig sei und diese Schulform erst dann wirklich bedroht sei, wenn man seine »Uneinigkeit nicht mehr ausdrücken könne oder wolle«. ${ }^{11}$ Ich kann Christensen nur zustimmen, was die Fähigkeit der folkehøjskoler anbelangt, vielfältige Meinungen und Erscheinungen offen zu diskutieren. Wenn aber, wie ich es oft beobachtet habe, die Streitkultur zu einer Tugend an sich hochstilisiert wird, wirkt sie selbstzerstörerisch.

Die folkehøjskoler und mit ihnen die Freien Schulen insgesamt stehen an einem Scheideweg. Niemals zuvor in ihrer Geschichte wurden ihre institutionelle, ihre ethische und ihre pädagogische Grundlage gleichzeitig so kritisch hinterfragt. Die Schulen müssen erkennen, daß sie ihre Freiheit auf Dauer nur bewahren können, wenn sie gemeinsam eine innovative Rolle in der gesellschaftlichen Diskussion um eine Neubestimmung des dänischen Nationalstaatsgedankens anstreben. Sie müssen außerdem erkennbar machen, wie sie die Idee des gemeinsamen Besten im Interesse der Allgemeinheit auch in Zukunft über den Weg der "folkelig oplysning « in die gesellschaftliche Realität Dänemarks übertragen wollen. Meiner Meinung nach bedeutet dies nicht unbedingt eine Entscheidung für oder gegen die Tradition oder die Moderne. Denn die tägliche Arbeit der Freien Schulen zeigt, daß die Traditionen der Grundtvigschen Pädagogik und seiner Aufklärungsphilosophie bis heute überzeugend auf ihre Schüler wirken. Was die Fachlichkeit angeht, haben die folkehøjskoler schon immer verstanden, sich flexibel auf die jeweils als modern geltenden Fächer einzustellen. 
In der Bewertung der »folkelighed" aber müssen sich die Freien Schulen von den Fesseln überkommener Traditionen lösen und sich modernen Ideen offensiv stellen. Erster und notwendiger Schritt in eine solche Offensive wäre der Versuch aller Freien Schulen auf eigene Initiative über einen längeren Zeitraum eine gemeinsame Erklärung zu erarbeiten. Diese Erklärung müßte die historischen Gemeinsamkeiten und Unterschiede der Freien Schulen darlegen, eine kritische Bestandsaufnahme ihrer gegenwärtigen Verfassung beinhalten und schließlich gemeinsame zukünttige Zielsetzungen aufzeigen. Die im Jahr 1999 begonnenenVartovsamtaler betrachte ich als eine erste, vorsichtige Initiative in diese Richtung. Bis weitere Schritte folgen, müssen die folkehøjskoler und die übrigen Freien Schulen noch oft über ihre eigenen Schatten springen.

Die folgende Geschichte kann als Beleg dafür genommen werden, daß der richtige Weg dabei zwischen der Tradition und der Moderne liegt. 1937 schrieb der Architekt und Schriftsteller Poul Henningsen in einem Feuilleton der Zeitung »Politiken« über Vartov: »Für mich gibt es keinen Zweifel, daß Vartov fallen und durch ein modernes Gebäude ersetzt werden sollte. Wir werden noch erleben, daß die praktische und raumwirtschaftliche Situation des Rathausplatzes dahin führt, daß es fallen muß«. ${ }^{12}$ Proteste gegenüber solch radikalen Erneuerungsplänen und der Wille, über Jahrhunderte gewachsene Substanz zu erhalten, verhinderten den Abriß. 1946 gelang es Kirkeligt Samfund das Gebäude zu erwerben und zuletzt in den 1990ern einer gründlichen Modernisierung zu unterziehen. Heute ist das traditionsreiche Haus mit seinem Grundtvigschen Geist nicht mehr aus der Mitte der modernen Metropole Kopenhagen wegzudenken.

\section{Noten}

1. Das Wort »folkehøjskole« wird auf deutsch korrekt mit »Heimvolkshochschule « übersetzt. Mit diesem Begriff werden auch die deutschen Varianten der dänischen folkehøjskoler bezeichnet. Da sie aber eine andere Struktur und andere Zielsetzungen haben als ihre dänischen Gegenstücke, möchte ich in meinem Text am dänischen Wort festhalten.

2. Es sei an dieser Stelle bemerkt, daß mir bewußt ist, daß es vielfältige Unterschiede zwischen den verschiedenen Formen der sog. „Freien Schulen« in Dänemark gibt. Ich nenne im vorliegenden Artikel folkehøjskoler und Freie Schulen oft zusammen, ohne auf diese Unterschiede aufmerksam $\mathrm{zu}$ machen, weil es um gemeinsame Faktoren geht, wie z.B. die historischen Wurzeln oder eine bestimmte Politik des Staates gegenüber dieser Schulform an sich. 
3. Ole Vig Jensen, Folkehøjskoler og »folkets almindelige anliggender«, in: Højskolebladet 26 (1993), S. 407-409; außer den hier zitierten Werken und Zeitschriftenartikeln habe ich nur eine kleine Anzahl von Handbüchern über Grundtvig und über die folkehøjskoler als Literaturgrundlage benutzt.

4. Lars Kaae, Ikkun som voxne Menneske-Børn: Grundtvig og frihed, in: Stykkevis og delt, Århus 1986, S. 82f., 86 u. 97.

5. N.F.S. Grundtvig, Folkeligheden, in: Danskeren, Bd. I (30.08.1848), S. 381-384.

6. Ove Korsgaard, Kampen om lyset, 1997, S. 191ff., 200f.

7. Morten Kvist, Ortodoksi og kætteri, in: Højskolebladet 28 (1994), S. 432.

8. K.E. Bugge, Vilhelm Nielsen (Red.), N.F.S. Grundtvig, Statsmæssig Oplysning - et udkast om samfund og skole, 1983, S. 15-19.

9. Regner Birkelund, Det grundtvigske og det rationalistiske oplysningsbegreb, in: Kvan 55 (1999), S. 22f.

10. Ebd., S. $24 f$.

11. Frederik Christensen, Det dobbelte spor, in: Højskolebladet 11 (1995), S. 163-167.

12. Hier zitiert und übersetzt nach einem Artikel von Jes Fabricius Møller, Min tid på Vartov, in: Vartovkollegiet 1949-1999, hrsg. vom Vartovkollegiet 1999, S. 44. 